\title{
Dielectric properties of benzylamine in 1,2,6-hexanetriol mixture using time domain reflectometry technique
}

\author{
M. B. Swami* ${ }^{\circledR}$, P. G. Hudge ${ }^{\dagger}$ and V. P. Pawar \\ *Department of Physics and Electronics, Maharashtra Udyagiri Mahavidyalaya \\ Udgir, Maharashtra, India \\ †Department of Physics, South Indian Children's Education Society's Degree College of Arts \\ Science and Commerce, Ambarnath (W), Maharashtra, India \\ \$rincipal, Sundarao Solanke Mahavidyalaya, Majalgaon, Maharashtra, India \\ \$zungaswami@gmail.com
}

Received 8 October 2016; Revised 14 November 2016; Accepted 15 November 2016; Published 16 December 2016

\begin{abstract}
The dielectric properties of binary mixtures of benzylamine-1,2,6-hexantriol mixtures at different volume fractions of 1,2,6hexanetriol have been measured using Time Domain Reflectometry (TDR) technique in the frequency range of $10 \mathrm{MHz}$ to $30 \mathrm{GHz}$. Complex permittivity spectra were fitted using Havriliak-Negami equation. By using least square fit method the dielectric parameters such as static dielectric constant $\left(\varepsilon_{0}\right)$, dielectric constant at high frequency $\left(\varepsilon_{\infty}\right)$, relaxation time $\tau$ (ps) and relaxation distribution parameter $(\beta)$ were extracted from complex permittivity spectra at $25^{\circ} \mathrm{C}$. The intramolecular interaction of different molecules has been discussed using the Kirkwood correlation factor, Bruggeman factor. The Kirkwood correlation factor $\left(g_{f}\right)$ and effective Kirkwood correlation factor $\left(g^{\text {eff }}\right)$ indicate the dipole ordering of the binary mixtures.
\end{abstract}

Keywords: Dielectric properties; time domain reflectometry; Kirkwood correlation factor; Bruggeman factor.

\section{Introduction}

In benzylamine $\left[\mathrm{C}_{6} \mathrm{H}_{5} \mathrm{CH}_{2} \mathrm{NH}_{2}\right]$ a benzyl group $\left[\mathrm{C}_{6} \mathrm{H}_{5} \mathrm{CH}_{2}\right]$ is attached to an amine functional group. This colorless liquid is a common precursor in organic synthesis. In the presence of moisture, benzylamine may weakly corrode some metals. It is used to make pharmaceuticals and other chemicals. Amines have a wide range of chemical, biological, pharmaceutical, industrial and condensed matter physics applications. Since amines and alcohols have the H-bond sites which can enter into intra-and intermolecular hydrogen bonding, it result into different conformations. ${ }^{1}$

Dielectric Relaxation Spectroscopy (DRS) technique is a powerful technique to study the intra- and intermolecular interactions in hydrogen bonded liquid system such as alcohols. In alcohol compounds, the hydroxyl $(-\mathrm{OH})$ group is attached to waterlogged hydrogen atom and these hydroxyl groups are classified as monohydric, dihydric, trihydric and polyhydric alcohols. Polyhydric alcohol is the hydrogen bonded liquid, where each molecule has two or more $-\mathrm{OH}$ groups. The $\mathrm{O}-\mathrm{H}$ bond in alcohol is highly polar. Due to this, hydrogen bonding requires a great agreement of energy in the form of heat to overcome these attractive forces.

In general, polyhydric alcohols exhibit the glass transition at temperature $(T g)$ relatively higher than those of usual molecular liquids with similar molecular size. ${ }^{2} 1,2$, 6-hexanetriol is the polyhydric alcohol, which consists of linear back bone chain with $\mathrm{OH}$ groups attached to every carbon atoms with aliphatic form. In this network, the reorientational dynamics of $\mathrm{OH}$ group is strong in the system. The number of $\mathrm{OH}$ groups per molecules is considered to play an important role on their systematic behavior. The $\mathrm{OH}$ groups mainly characterizing the liquid are dielectrically active. ${ }^{2,3}$ The dielectric properties of triols have attracted the attention of many researchers. ${ }^{2-4}$ The structural information about 1,2,6-hexanetriol in liquid state has been extensively studied through dielectric relaxation measurements. ${ }^{5}$ The constructive technique of Time Domain Reflectometry (TDR) is used to study the dielectric relaxation parameters of liquids. $^{6-9}$ Significant dielectric relaxation work has been done in our laboratory on diol and 2-butoxyethanol in water mixtures using TDR. ${ }^{10,11}$ Pawar and Patil have studied temperature-dependent dielectric relaxation on molecular interaction in binary mixture of dimethylene chloride with n-methyl formamide using TDR technique. ${ }^{12}$ Much research work has been carried out on dielectrics of liquids such as alcohols, bio-polymers, etc. ${ }^{13-20}$

The key point of these studies were to understand the structure of benzylamine in its pure and alteration of benzylamine in 1,2,6-hexanetriol. The present work deals with time domain dielectric relaxation study of benzylamine in 1,2,6hexanetriol at $25^{\circ} \mathrm{C}$ in the frequency range of $10 \mathrm{MHz}$ to

This is an Open Access article published by World Scientific Publishing Company. It is distributed under the terms of the Creative Commons Attribution 4.0 (CC-BY) License. Further distribution of this work is permitted, provided the original work is properly cited. 
$30 \mathrm{GHz}$. From the measured complex permittivity spectra, static dielectric constant $\left(\varepsilon_{0}\right)$, dielectric constant at high frequency $\left(\varepsilon_{\infty}\right)$, relaxation time $\tau$ (ps) and relaxation distribution parameter $(\beta)$ were obtained by least square fit method. The Kirkwood correlation factor and Bruggeman factor were determined.

\section{Experimental Procedure}

\subsection{Materials and measurements}

The benzylamine (Benzyl) and 1,2,6-hexanetriol (Hex) were obtained commercially from S. D. Fine Chemical Limited, India and was used without further purification. The solutions were prepared very carefully at different volume fractions of benzylamine in 1,2,6-hexanetriol. In order to avoid the uptake of moisture from air (or) evaporation of a nonvolatile solvent tightly packed flasks are used.

The dielectric spectra were obtained by the TDR technique. $^{21,22}$ The Tektronix model no. DSA8200 Digital Serial Analyzer sampling mainframe along with the sampling module 80E08 has been used for the TDR. Figure 1 shows the experimental setup based on the TDR technique. A repetitive fast rise pulse with $18 \mathrm{ps}$ incident pulse rise time and $20 \mathrm{ps}$ reflected pulse rise time was fed through coaxial line system impedance of $50 \mathrm{ohm}$. All measurements are carried out in open load condition.

Sampling oscilloscope monitors changes in step pulse after reflection from the end of line. Reflected pulse without sample $R_{1}(t)$ and with sample $R_{x}(t)$ were recorded in time window of $5 \mathrm{~ns}$ and digitized in 2000 points in the memory of the oscilloscope and transferred to the Computer. The rise time TDR 18 ps corresponds to bandwidth $17.69 \mathrm{GHz}$ (TDR bandwidth $\approx 1 /(\pi \times$ rise time $)) .{ }^{11}$ The selection of proper time window, sampling rate and bilinear calibration method suggested by Cole et al. were useful to measure the accurate complex permittivity at higher frequencies. ${ }^{7}$ The smaller time window causes loss of signal while larger time window includes unwanted reflections. Thus selection of proper time window is important to minimize these effects. Further the

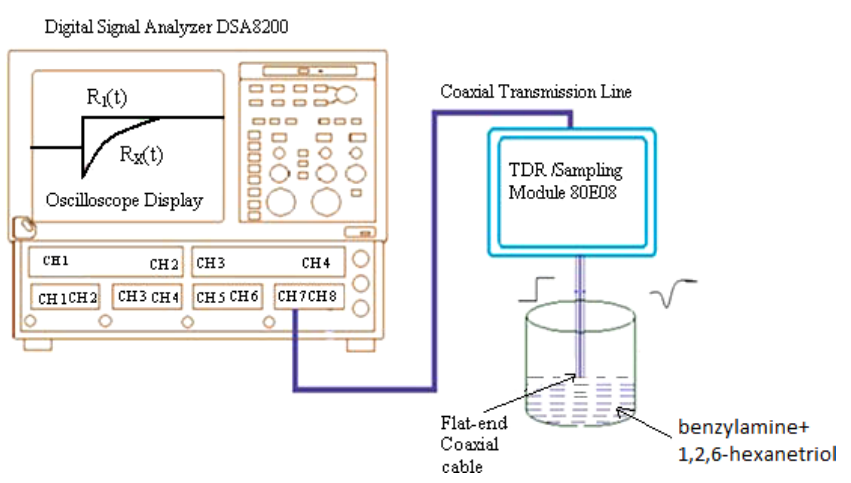

Fig. 1.

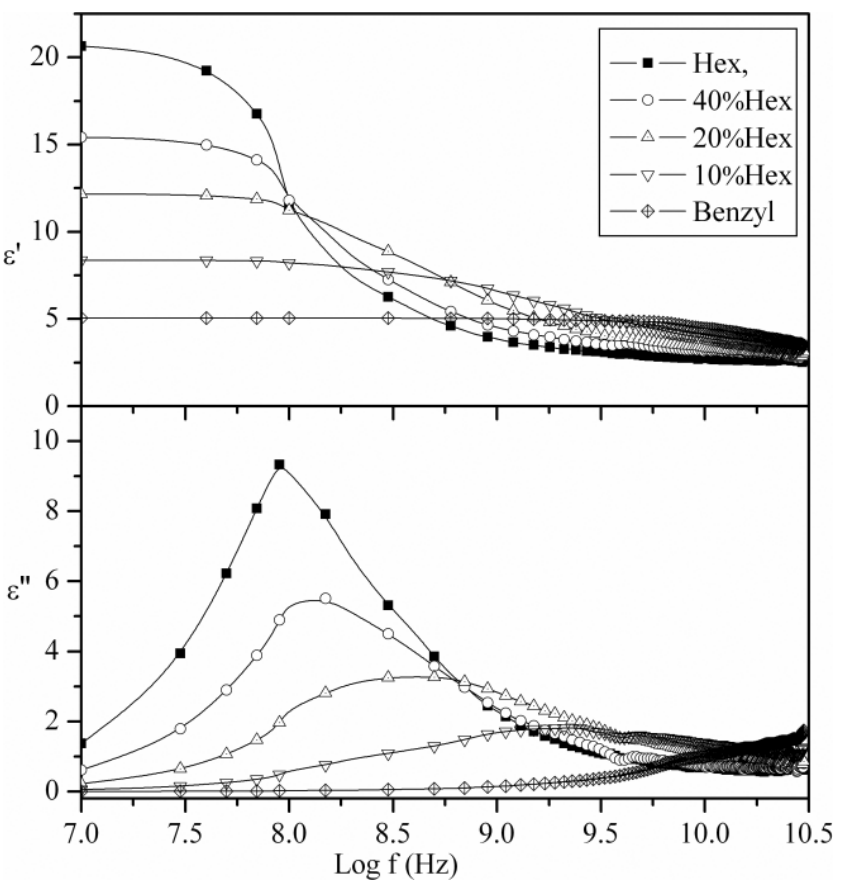

Fig. 2. Complex permittivity spectra of benzylamine-1,2,6-hexanetriol mixtures at $25^{\circ} \mathrm{C}$.

Fourier transformation of the pulses and data analysis were done earlier to determine complex permittivity spectra $*(\omega)$ using nonlinear least squares fit method. ${ }^{7,9}$

The dielectric permittivity $\varepsilon^{\prime}$ and dielectric loss $\varepsilon^{\prime \prime}$ of benzylamine $+1,2,6$-hexanetriol mixtures at $25^{\circ} \mathrm{C}$ were measured using TDR technique as shown in Fig. 2. From Fig. 2 it is observed that the real part of complex permittivity spectrum of benzylamine $+1,2,6$-hexanetriol mixtures is almost independent of frequency below $0.1 \mathrm{GHz}$. At the relaxation frequency the oscillation of the applied field becomes very fast for the molecules to have time to fully rotate. As a result the real part $\varepsilon^{\prime}$ from the complex permittivity will decrease when the relaxation frequency is reached since less of the applied field is canceled by the dipoles as the frequency is increased. In an imaginary part $\varepsilon^{\prime \prime}$ from the complex permittivity spectra the peak value occurs at this frequency since most of the energy is lost at that point. The values of $\varepsilon^{\prime}$ decreases with decrease in concentration of 1,2,6-hexanetriol and the peak value of dielectric loss max shifts towards higher frequencies which indicates decrease in relaxation time.

\section{Result and Discussion}

Recently, dielectric measurements were performed on water + 2-butoxyethanol mixture using TDR technique. ${ }^{11}$ It is observed that the complex permittivity for water + 2-butoxyethanol mixture is an asymmetric shape i.e., the deviation of the dielectric loss peak occurs for these systems 
from the Cole-Davidson function at high frequency. ${ }^{11}$ It is mathematically proved that asymmetric distribution of relaxation time is associated with the cooperative mechanism of dielectric relaxation. ${ }^{23}$ Therefore, for the evaluation of dielectric parameters, the frequency-dependent complex permittivity $\left[\varepsilon^{*}(\omega)\right]$ spectra were fitted in Havriliak-Negami $(\mathrm{HN})$ equation using least squares fit method. ${ }^{24}$

$$
\varepsilon^{*}(\omega)=\varepsilon_{\infty}+\frac{\varepsilon_{0}-\varepsilon_{\infty}}{\left[1+(j \omega \tau)^{1-\alpha}\right]^{\beta}},
$$

where $\varepsilon_{0}$ is the static permittivity, $\varepsilon_{\infty}$ is the permittivity at high frequency, $\tau$ is the relaxation time in picosecond, $\alpha$ and $\beta$ are the empirical parameters for the distribution of relaxation times with values between 0 and 1 . The $\mathrm{HN}$ equation includes three relaxation models. The Debye model ${ }^{25}(\alpha=0$ and $\beta=1)$ shows the single relaxation whereas the Cole-Cole ${ }^{26}$ $(0 \leq \alpha \leq 1$ and $\beta=1)$ and Cole-Davidson ${ }^{27}(\alpha=0$ and $0 \leq \beta \leq 1$ ) models suggest the symmetric and asymmetric distribution of relaxation times. The dielectric relaxation parameters $\left(\varepsilon_{0}, \varepsilon_{\infty}, \tau\right.$ and $\left.\beta\right)$ for benzylamine-1,2, 6-hexantriol mixtures with volume fraction of $1,2,6$-hexanetriol at $25^{\circ} \mathrm{C}$ are listed in Table 1.

Numbers in bracket denotes uncertainties in the last significant digits obtained by the least square fit method. For example, $4.33(2)$ means $4.33 \pm 0.02$ and 1470.54(34) means $1470.54 \pm 0.34$.

The higher values of relaxation time in the rich region of 1,2,6-hexnaetriol suggest that there is cooperative relaxation with larger hindrance. The value of relaxation time for pure 1,2,6-hexanetriol has agreed with the reported value in Ref. 16.

\subsection{Kirkwood correlation factor}

The Kirkwood correlation factor ' $g$ ' is also parameter containing information regarding orientation of electric dipoles in polar liquids. ${ }^{28}$ The value of ' $g$ ' forpure liquid is given by expression

$$
g \mu^{2} \frac{4 \pi N \rho}{9 k T M}=\frac{\left(\varepsilon_{0}-\varepsilon_{\infty}\right)\left(2 \varepsilon_{0}+\varepsilon_{\infty}\right)}{\varepsilon_{0}\left(\varepsilon_{\infty}+2\right)^{2}},
$$

where ' $\mu$ ' dipole moment is in gas phase, ' $N$ ' is Avogadro's number, ' $M$ ' is molecular weight, ' $\rho$ ' is the density of the liquid and ' $k T$ ' has usual meaning. We have taken $\varepsilon_{\infty}$ value from refractive index $\left(\varepsilon_{\infty}=n^{2}\right)$ data at $25^{\circ} \mathrm{C}$.

For binary mixture, the static dielectric permittivity needs to be considered as the dipole orientation correlation factor ( $\left.g^{\text {eff }}\right)$. The modified form of Eq. (3) is used to study the orientation of the electric dipoles in the binary mixtures as follows. $^{28}$

$$
\begin{aligned}
& \frac{4 \pi N}{9 k T}\left[\frac{\mu_{\mathrm{HEX}}^{2} \rho_{\mathrm{HEX}} V_{\mathrm{HEX}}}{M_{\mathrm{HEX}}}+\frac{\mu_{A}^{2} \rho_{A}\left(1-V_{\mathrm{HEX}}\right)}{M_{A}}\right] \times g^{\mathrm{eff}} \\
& =\frac{\left(\varepsilon_{0 m}-\varepsilon_{\infty m}\right)\left(2 \varepsilon_{0 m}+\varepsilon_{\infty m}\right)}{\varepsilon_{0 m}\left(\varepsilon_{\infty m}+2\right)^{2}}
\end{aligned}
$$

where $M_{\mathrm{HEX}}$ and $M_{A}$ are molecular weight of 1,2,6-hexanetriol and benzylamine, respectively. $\rho_{\mathrm{HEX}}$ and $\rho_{A}$ are corresponding densities. $V_{\mathrm{HEX}}$ is volume fraction of 1,2 , 6-hexanetriol in benzylamine. $\varepsilon_{0 m}$ and $\varepsilon_{\infty m}$ are the static dielectric constant and dielectric constant at high frequency of the mixtures. To calculate the values of $g^{\text {eff }}$, we have taken $\mu=1.38$ and $2.73 \mathrm{D}^{29}$ for benzylamine and 1,2,6hexanetriol, respectively. ${ }^{30}$ The Kirkwood correlation factor $g^{\text {eff }}$ for benzylamine-1,2,6-hexanetriol mixtures at different volume fractions of 1,2,6-hexanetriols are shown in Table 2.

The observed $g^{\text {eff }}$ value is greater than unity in benzylamine-1,2,6-hexanetriol mixtures, which suggests that the heterogeneous interaction between the molecules of the constituent polar liquids and in such way that to form multimers with increasing parallel orientation of electric dipoles.

\subsection{Bruggeman factor}

The static permittivity of two-component mixture must lie somewhere between two extremes corresponding to static permittivity of two liquids. The Bruggeman mixture formula has been proposed to understand the dipole interaction in the mixture of two liquids. ${ }^{31}$ This formula state that static permittivity of the mixture $\left(\varepsilon_{0}\right)_{m}$, benzylamine $\left(\varepsilon_{0}\right)_{A}$, and 1,2,6-hexanetriol $\left(\varepsilon_{0}\right)_{\mathrm{HEX}}$ can be related to volume fraction of 1,2,6-hexanetriol $\left(V_{\mathrm{HEX}}\right)$ in the mixture as

$$
f_{B}=\left(\frac{\varepsilon_{0 m}-\varepsilon_{0 \mathrm{HEX}}}{\varepsilon_{0 A}-\varepsilon_{0 \mathrm{HEX}}}\right)\left(\frac{\varepsilon_{0 A}}{\varepsilon_{0 m}}\right)^{1 / 3}=1-V_{H E X} .
$$

Table 1. Dielectric parameters for benzylamine-1,2,6-hexanetriol mixture at $25^{\circ} \mathrm{C}$.

\begin{tabular}{lcccl}
\hline$V_{\text {HEX }}$ & $\varepsilon_{0}$ & $\tau(\mathrm{ps})$ & $\varepsilon_{\infty}$ & \multicolumn{1}{c}{$\beta$} \\
\hline 0 & $4.33(2)$ & $8.62(3)$ & $2.01(2)$ & 1.000 \\
0.1 & $8.70(2)$ & $65.59(81)$ & $2.24(7)$ & $0.624(3)$ \\
0.2 & $12.83(5)$ & $331.79(54)$ & $2.78(1)$ & $0.723(3)$ \\
0.4 & $15.05(11)$ & $866.55(19)$ & $3.03(1)$ & $0.815(6)$ \\
1 & $19.30(19)$ & $1470.54(34)$ & $2.58(2)$ & $0.884(8)$ \\
\hline
\end{tabular}

Table 2. Kirkwood correlation factor $g^{\text {eff }}$ for benzylamine1,2,6-hexanetriol mixtures with volume fraction of 1,2,6-hexanetriol $\left(V_{\mathrm{HEX}}\right)$ at $25^{\circ} \mathrm{C}$.

\begin{tabular}{lc}
\hline$V_{\mathrm{HEX}}$ & $g^{\text {eff }}$ \\
\hline 0.0 & $1.49(9)$ \\
0.1 & $1.72(10)$ \\
0.2 & $2.51(10)$ \\
0.4 & $3.21(16)$ \\
1.0 & $5.26(11)$ \\
\hline
\end{tabular}




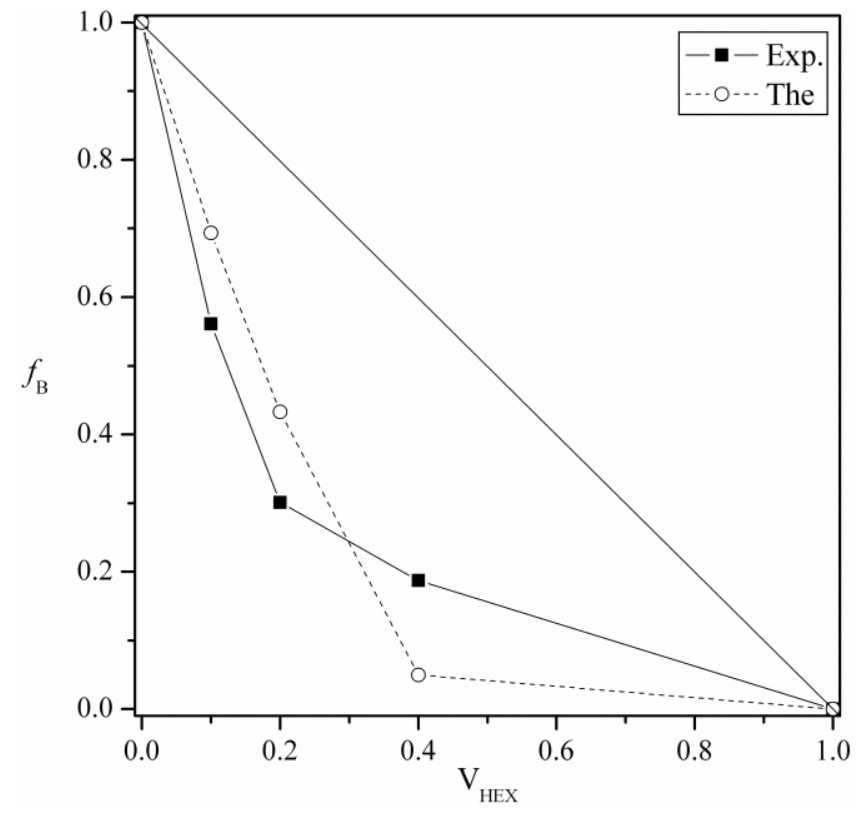

Fig. 3. Bruggeman factor $\left(f_{B}\right)$ versus volume fraction of $1,2,6-$ hexanetriol $\left(V_{\mathrm{HEX}}\right)$ at $25^{\circ} \mathrm{C}$.

The Bruggeman expression predicts a linear relationship between $f_{\mathrm{B}}$ and the volume fraction of 1,2,6-hexanetriol ( $V_{\mathrm{HEX}}$ ) but the experimental values of $f_{B}$ shows a nonlinear behavior as shown in Fig. 3. To explain the nonlinear relationship Eq. (3) is modified as follows. ${ }^{31}$

$$
\begin{aligned}
f_{B} & =\left(\frac{\varepsilon_{0 m}-\varepsilon_{0 \mathrm{HEX}}}{\varepsilon_{0 A}-\varepsilon_{0 \mathrm{HEX}}}\right)\left(\frac{\varepsilon_{0 A}}{\varepsilon_{0 m}}\right)^{1 / 3} \\
& =1-\left[a-(a-1) V_{H E X}\right] V_{H E X},
\end{aligned}
$$

where ' $a$ ' is arbitrary parameter, the value of $a=1$ means the ideal mixture with no interaction between solute and solvent. In Fig. 2 solid line represents the ideal behavior according to Eq. (4). Experimental values represent the deviation from ideal behavior of mixture which shows the solute-solvent molecular interaction between benzyalmine1,2,6-hexanetriol mixtures. The calculated value of ' $a$ ' for benzyalmine-1,2,6-hexanetriol mixture is determined using least square fit method and it is found to be 3.29 at $25^{\circ} \mathrm{C}$.

\section{Conclusions}

The dielectric relaxation behavior of benzylamine-1,2,6hexantriol mixture have been studied using TDR technique in the frequency range $10 \mathrm{MHz}$ to $30 \mathrm{GHz}$ at $25^{\circ} \mathrm{C}$. The dielectric constant and relaxation time were determined using $\mathrm{HN}$ equation for benzylamine-1,2,6-hexantriol mixture. The orientation correlations between neighboring molecules due to hydrogen bonding interaction are determined in terms of Kirkwood factors. The modified Bruggeman equation is well fitted with experimental dielectric constant.

\section{Acknowledgments}

The Department of Science and Technology, New Delhi is gratefully acknowledged (project No.SR/S2/LOP-25/2007) for laboratory facility. We are thankful to Prof. S.C. Mehrotra, Ramanujan Chair Professor, Department of Computer Science and IT, Dr. B.A.M. University, Aurangabad and Prof. A. C. Kumbharkhane, School of Physical Sciences, S.R. T.M. University, Nanded for the fruitful discussion. We also thank Prof. G. N. Shinde for his helpful suggestions.

\section{References}

${ }^{1}$ M. B. Swami, V. P. Pawar, P. G. Hudge and A. C. Kumbharkhane, Dielectric relaxation study of amines in 2,3-butanediol mixture using picosecond time domain reflectometry technique, J. Mol. Liq. 190, 178 (2014).

${ }^{2} \mathrm{~N}$. Masahiro and N. Ryusuke, Dynamics and structure of hydrogen-bonding glass formers: Comparison between hexanetriol and sugar alcohols based on dielectric relaxation, J. Phys. Rev. 81, 041501 (2010).

${ }^{3}$ P. Lunkenheimer, A. Pimenor, B. Schiener, R. Bohmer and A. Loidal, High-frequency dielectric spectroscopy on glycerol, Eur. Phys. Lett. 33, 611 (1996).

${ }^{4}$ V. I. Zhuravlev, N. V. Lifanova and T. M. Usacheva, Structure of some liquid alcohols, diols and triols: an analysis of dielectric data, J. Mol. Liq. 120, 107 (2005).

${ }^{5}$ N. V. Lifanova, T. M. Usacheva, V. I. Zhuravlev and V. K. Matveev, Dielectric properties of 2,5-hexanediol, Rus. J. Phys. Chem. 77, 477 (2003).

${ }^{6}$ J. B. Hasted, Aqueous Dielectric (Chapman and Hall, London, 1973).

${ }^{7}$ R. H. Cole, J. G. Berberian, S. Mashimo, G. Chryssikos, A. Burns and E. Tombari, Time domain reflection methods for dielectric measurements to $10 \mathrm{GHz}$, J. Appl. Phys. 66, 793 (1989).

${ }^{8}$ D. Bertolini, M. Cassettari, G. Salvetti, E. Tomabari and S. Versoni, Time domain reflectometry to study the dielectric properties of liquids: Some problems and solutions, Rev. Sci. Instrum. 62, 450 (1991).

${ }^{9}$ A. C. Kumbharkhane, S. M. Puranik and S. C. Mehrotra, Dielectric relaxation of tert-butyl alcohol-water mixtures using a time-domain technique, Faraday Trans. 87, 1569 (1991).

${ }^{10}$ S. D. Chavan, B. D. Watode, P. G. Hudge, D. B. Suryawanshi, C. G. Akode, A. C. Kumbharkhane and S. C. Mehrotra, Dielectric relaxation and hydrogen bond interaction study of diol-water mixtures, Ind. J. Phys. 84, 419 (2010).

${ }^{11}$ Y. S. Joshi and A. C. Kumbharkhane, Study of dielectric relaxation and hydrogen bonding in water+ 2-butoxyethanol mixtures using TDR technique, Fluid Phase Equilib. 317, 96 (2012).

${ }^{12}$ V. P. Pawar and A. V. Patil, (Dielectric relaxation studies on molecular interaction in binary mixture of dimethylene chloride with n-methylformamide), Fluid Phase Equilib. 376, 111 (2014).

${ }^{13}$ Y. S. Joshi, P. G. Hudge, A. C. Kumbharkhane and S. C. Mehrotra, The dielectric relaxation study of 2 (2-alkoxyethoxy) ethanolwater mixtures using time domain reflectometry, J. Mol. Liq. 163, 70 (2011).

${ }^{14}$ Y. S. Joshi and A. C. Kumbharkhane, Study of heterogeneous interaction in binary mixtures of 2-methoxyethanol-water using dielectric relaxation spectroscopy, J. Mole. Liq. 161, 120 (2011). 
${ }^{15}$ A. C. Kumbharkhane, M. N Shinde, S. C. Mehrotra, N. Oshiki, N. Shinyashiki and S. Yagihara, Structural behavior of alcohol - 1, 4-dioxane mixtures through dielectric properties using TDR, J. Phys. Chem. A 113, 10196 (2009).

${ }^{16}$ S. D. Shirgire, P. G. Hudge and A. C. Kumbharkhane, Temperature-dependent dielectric relaxation study of 1,2,6-hexanetriol using TDR method, Phys. Chem. Liq. 50, 316 (2012).

${ }^{17}$ P. G. Hudge, M. P. Lokhande and A. C. Kumbharkhane, The study of dielectric relaxation in aqueous carbohydrates solutions using time domain reflectometry technique, Indian J. Phys. 86, 813 (2012).

${ }^{18}$ S. S. Kadam, P. G. Hudge and A. C. Kumbharkhane, Dielectric relaxation study of 2 and 3-chloroaniline and 2 and 3-methoxyaniline with 1,4-dioxane mixtures using time domain technique, J. Mol. Liq. 177, 426 (2013).

${ }^{19}$ P. G. Hudge, D. S. Gond, R. N. Gacche, M. P. Lokhande and A. C. Kumbharkhane, Dielectric relaxation study of DNA in aqueous solution using time domain reflectometry, Ind. J. Phys. 87, 543 (2013).

${ }^{20}$ M. B. Swami, V. P. Pawar, P. G. Hudge and A. C. Kumbharkhane, Dielectric relaxation study of amines in 2, 3-butanediol mixture using picosecond time domain reflectometry technique, J. Mol. Liq. 190, 178 (2014).

${ }^{21}$ R. B. Talware, P. G. Hudge, Y. S. Joshi and A. C. Kumbharkhane, Dielectric relaxation study of glycine-water mixtures using time domain reflectometry technique, Phys. Chem. Liq. 50, 102 (2012).
${ }^{22}$ A. V. Patil, G. N. Shinde and V. P. Pawar, Dielectric relaxation study of hydrogen bonded structures in ethanolamine with diethanolamine using TDR technique, J. Mol. Liq. 168, 42 (2012).

${ }^{23}$ S. Havriliak and S. Negami, A complex plane representation of dielectric and mechanical relaxation processes in some polymers, Polymer 8, 161 (1967).

${ }^{24}$ S. Havriliak and S. Negami, A complex plane analysis of $\alpha$-dispersions in some polymer systems, J. Polym. Sci. C 14, 99 (1966).

${ }^{25}$ P. Debye, Polar Molecules (The Chemical Catalogue Company, New York, 1929).

${ }^{26}$ K. S. Cole and R. H. Cole, Dispersion and absorption in dielectrics I. Alternating current characteristics, J. Chem. Phys. 9, 341 (1941).

${ }^{27}$ D. W. Davidson and R. H. Cole, Dielectric relaxation in glycerine, J. Chem. Phys. 18, 1417 (1950).

${ }^{28}$ J. G. Kirkwood, The dielectric polarization of polar liquids, J. Chem. Phys. 7, 911 (1939).

${ }^{29}$ T. M. Usacheva, V. I. Zhuravlev, N. V. Lifanova and V. K. Matveev, Relaxation processes of the structural rearrangement of clusters in liquids: 1,2-ethanediol, 1,2-propanediol, and 1,2,6hexanetriol, J. Struct. Chem. 52, 1153 (2011).

${ }^{30}$ D. R. Lide (ed.), CRC Handbook of Chemistry and Physics, 87 th edn. (CRC Press, 2006).

${ }^{31}$ D. A. G. Bruggeman, Calculation of various physical constants of heterogeneous substances. I. Dielectric constants and conductivities of the mixed body of isotropic substances, Ann. Phys. 416, 636 (1935). 